\title{
CHEMICAL BINDING EFFECTS IN RESONANCE- POTENTIAL INTERFERENCE SCATTERING FOR HARMONIC CRYSTALS
}

\author{
A. Kuwaifi and G. C. Summerfield \\ The Department of Nuclear Engineering, The University of Michigan, Ann Arbor, MI 48109, U.S.A.
}

(Received 29 August 1990)

\begin{abstract}
The neutron scattering cross section which is the quantity directly measured in experiments is given by the absolute square of the scattering amplitude. For energies near a resonance, this yields three terms : potential, resonant and interference. In this paper, we deal with the interference neutron scattering cross section which is written in terms of a three-point correlation function. This function is calculated for the ideal gas and harmonic crystal models. For short collision times, the interference result for harmonic crystals is the same as the ideal gas but it has an effective temperature. This is the same effective temperature as was previously found for absorption and pure resonant processes. Therefore, the interference scattering cross section can be treated in the same way resonant scattering and absorption are treated using an ideal gas result with the usual effective temperature.
\end{abstract}

\section{INTRODUCTION}

Resonant capture and scattering are important processes for neutron interactions. For a single isolated resonance and for a free atom at rest, the cross section was given by Breit and Wigner (1936). If the atoms have a velocity distribution described by the Maxwell-Boltzmann law, the cross section is given by Bethe and Placzeck (1937). Lamb (1939) was the first to discuss and calculate the absorption line shape for an atom bound in a crystal lattice.

Elastic scattering is composed of three terms: (a) a resonance term; (b) an interference term; and (c) a potential term. Potential scattering dominates at energies far from resonances, and resonant scattering dominates near a resonance peak. Since these scattering processes are coherent an additional interference term arises between these two limits. Van Hove (1954) introduced a two-point correlation function related to potential scattering. Word and Trammel (1981) showed that the cross section for the elastic scattering of neutrons at resonance from nuclei bound in a crystal is related to a four-point correlation function.

Shamaoun and Summerfield (1990) calculated the resonant differential scattering cross section for harmonic crystals to examine the chemical binding effect for resonant scattering. Placzek and Van Hove (1955) have shown that interference scattering becomes negligible at large neutron energies but it must be included at very low neutron energies. In this paper we calculate the interference scattering cross section which can be written in terms of a three-point correlation function. For harmonic crystals, we calculate that function to see the chemical binding effect on interference scattering. We show that, for harmonic crystals and short collision times, interference scattering can be treated using the ideal gas result with the usual effective temperature.

\section{GENERAL FORMULATION FOR NEUTRON SCATTERING}

The starting point of our work is the formula given in two books by (Osborn and Yip, 1966; Lovesey and Springer, 1977) which give the differential scattering cross section for thermal neutrons:

$$
\frac{\mathrm{d}^{2} \sigma}{\mathrm{d} \epsilon \mathrm{d} \Omega}=\sum_{\mathrm{i}, \mathrm{f}}\left(\frac{E_{\mathrm{f}}}{E_{\mathrm{i}}}\right)^{1 / 2} g\left(\varepsilon_{\mathrm{i}}\right)\left|\frac{m}{2 \pi \hbar^{2}}\langle f|T| i\rangle\right|^{2} \delta\left(E_{\mathrm{i}}+\varepsilon_{\mathrm{i}}-E_{\mathrm{f}}-\varepsilon_{\mathrm{f}}\right),
$$

where $\epsilon$ is the energy transfer: 


$$
\epsilon=E_{\mathrm{f}}-E_{\mathrm{i}}=\frac{\hbar^{2}\left(K_{\mathrm{f}}^{2}-K_{\mathrm{i}}^{2}\right)}{2 m} .
$$

$E_{\mathrm{i}}$ and $E_{\mathrm{f}}$ are the initial and final neutron energies, respectively, $\varepsilon_{\mathrm{i}}$ and $\varepsilon_{\mathrm{f}}$ are the target energies and $m$ is the neutron mass. The $\delta$-function represents the conservation of energy, and the matrix $\langle f|T| i\rangle$ can be approximated for resonant and potential scattering as :

$$
\langle f|T|\rangle=\langle f|V| i\rangle+\sum_{j} \frac{\langle f|V| j\rangle\langle j|V| i\rangle}{E_{\mathrm{i}}-E_{0}+\varepsilon_{\mathrm{i}}-\varepsilon_{j}+i \frac{\Gamma(j)}{2}},
$$

where $|i\rangle,|j\rangle$ and $|f\rangle$ are the initial, intermediate and final states, respectively (more detail is given by Shamaoun and Summerfield, 1990). Here the neutron level width is taken to be independent of the intermediate state. The validity of this has been shown by Kuwaifi et al. (1990). The matrix elements $\langle f|V| j\rangle$ and $\langle j|V| i\rangle$ are given by :

$$
\begin{aligned}
& \langle f|V| j\rangle=M_{\mathrm{c}}\left\langle f\left|\exp \left(-i \bar{K}_{\mathrm{f}} \cdot \bar{R}_{L}\right)\right| j\right\rangle, \\
& \langle j|V| i\rangle=M_{\mathrm{c}}^{*}\left\langle j\left|\exp \left(-i \bar{K}_{\mathrm{i}} \cdot \bar{R}_{L}\right)\right| i\right\rangle,
\end{aligned}
$$

where $K_{\mathrm{i}}$ and $K_{\mathrm{f}}$ are the neutron's initial and final wave vectors, $\bar{R}_{L}$ is the position of the $L$ th nucleus in the laboratory system and $M_{\mathrm{c}}$ and $M_{\mathrm{c}}^{*}$ are the purely nuclear matrix elements.

The square of the absolute value of the $T$-matrix in equation (3) gives:

$$
|\langle f|T| i\rangle|^{2}=|\langle f|V| i\rangle|^{2}+\left|\sum_{j} \frac{\langle f|V| j\rangle\langle j|V| i\rangle}{E_{\mathrm{i}}-E_{0}+\varepsilon_{\mathrm{i}}-\varepsilon_{j}+i \frac{\Gamma}{2}}\right|^{2}+2 \operatorname{Re} \sum_{j}\langle i|V| f\rangle \frac{\langle f|V| j\rangle\langle j|V| i\rangle}{E_{\mathrm{i}}-E_{0}+\varepsilon_{\mathrm{i}}-\varepsilon_{j}+i \frac{\Gamma}{2}} .
$$

Substituting equation (6) into (1) we get :

$$
\frac{\mathrm{d}^{2} \sigma_{n, n}}{\mathrm{~d} \epsilon \mathrm{d} \Omega}=\frac{\mathrm{d}^{2} \sigma_{p}}{\mathrm{~d} \epsilon \mathrm{d} \Omega}+\frac{\mathrm{d}^{2} \sigma_{n, n_{0}}}{\mathrm{~d} \epsilon \mathrm{d} \Omega}+\frac{\mathrm{d}^{2} \sigma_{\text {int }}}{\mathrm{d} \epsilon \mathrm{d} \Omega},
$$

where

$$
\begin{gathered}
\frac{\mathrm{d}^{2} \sigma_{p}}{\mathrm{~d} \epsilon \mathrm{d} \Omega}=\sum_{i, f}\left(\frac{E_{\mathrm{f}}}{E_{\mathrm{i}}}\right)^{1 / 2} g\left(\varepsilon_{\mathrm{i}}\right)\left(\frac{m}{2 \pi \hbar^{2}}\right)^{2}|\langle f|V| i\rangle|^{2} \delta\left(-\epsilon+\epsilon_{\mathrm{i}}-\varepsilon_{\mathrm{f}}\right), \\
\frac{\mathrm{d}^{2} \sigma_{n, n_{0}}}{\mathrm{~d} \epsilon \mathrm{d} \Omega}=\sum_{\mathrm{i}, f}\left(\frac{E_{\mathrm{f}}}{E_{\mathrm{i}}}\right)^{1 / 2} g\left(\varepsilon_{\mathrm{i}}\right)\left(\frac{m}{2 \pi \hbar^{2}}\right)^{2}\left|\sum_{j} \frac{\langle f|V| j\rangle\langle j|V| i\rangle}{E_{\mathrm{i}}-E_{0}+\varepsilon_{\mathrm{i}}-\varepsilon_{j}+i \frac{\Gamma}{2}}\right|^{2} \delta\left(-\epsilon+\varepsilon_{\mathrm{i}}-\varepsilon_{\mathrm{f}}\right), \\
\frac{\mathrm{d}^{2} \sigma_{\text {int }}}{\mathrm{d} \epsilon \mathrm{d} \Omega}=\sum_{\mathrm{i} . f}\left(\frac{E_{\mathrm{f}}}{E_{\mathrm{i}}}\right)^{1 / 2} g\left(\varepsilon_{\mathrm{i}}\right)\left(\frac{m}{2 \pi \hbar^{2}}\right)^{2} 2 \operatorname{Re} \sum_{j}\langle i|V| f\rangle \frac{\langle f|V| j\rangle\langle j|V| i\rangle}{E_{\mathrm{i}}-E_{0}+\varepsilon_{\mathrm{i}}-\varepsilon_{j}+i \frac{\Gamma}{2}} \delta\left(-\epsilon+\varepsilon_{\mathrm{i}}-\varepsilon_{\mathrm{f}}\right) .
\end{gathered}
$$

As we see, the elastic scattering cross section is composed of three factors the first term represents a potential scattering which gives the "hard sphere" contribution, and the second term represents the resonance elastic scattering due to the re-emission of the neutron. Since these scattering processes are coherent an interference term arises. This term represents the interference between the resonance and potential scattering. Shamaoun and Summerfield (1990) examined the chemical binding effect for resonant scattering for harmonic crystals. In the following we will rederive the interference differential scattering cross section for harmonic crystals. Recently Trammell (1984) suggested that this interference term can be used to obtain new information about structures and motions of condensed-matter systems. 


\section{DERIVATION OF INTERFERENCE CORRELATION FUNCTION}

Starting from equation (10) and using :

$$
\frac{1}{E_{\mathrm{i}}-E_{0}+\varepsilon_{\mathrm{i}}-\varepsilon_{j}+i \frac{\Gamma}{2}}=i \int_{0}^{\infty} \frac{\mathrm{d} t}{\hbar} \exp \left[i\left(E_{\mathrm{f}}-E_{0}+\varepsilon_{\mathrm{f}}-\varepsilon_{j}+i \frac{\Gamma}{2}\right) \frac{t}{\hbar}\right]
$$

and

$$
\delta\left(-\epsilon+\varepsilon_{\mathrm{i}}-\varepsilon_{\mathrm{f}}\right)=\frac{1}{2 \pi} \int_{-\infty}^{+\infty} \frac{\mathrm{d} T}{\hbar} \exp \left[i\left(-\epsilon+\varepsilon_{\mathrm{i}}-\varepsilon_{\mathrm{f}}\right) \frac{T}{\hbar}\right] .
$$

Similarly to (4) and (5), we can write $\langle i|V| f\rangle$ as follows :

$$
\langle i|V| f\rangle=M_{\mathrm{c}}^{*}\left\langle i\left|\exp \left(i \Delta \bar{K} \cdot \bar{R}_{L}\right)\right| f\right\rangle,
$$

where

$$
\Delta \bar{K}=\bar{K}_{\mathrm{f}}-\bar{K}_{\mathrm{i}}
$$

Now substitute equations (4), (5) and (13) into equation (10) to get:

$$
\frac{\mathrm{d}^{2} \sigma_{\text {int }}}{\mathrm{d} \epsilon \mathrm{d} \Omega}=Q \operatorname{Re} i \int_{0}^{\infty} \frac{\mathrm{d} t}{\hbar} \exp \left[i\left(\Delta E+i \frac{\Gamma}{2}\right) \frac{t}{\hbar}\right] \int_{-\infty}^{+\infty} \frac{\mathrm{d} T}{\hbar} \exp \left(-i \epsilon \frac{T}{\hbar}\right) C
$$

where

$$
\begin{gathered}
Q=\frac{1}{\pi}\left(\frac{m}{2 \pi \hbar^{2}}\right)^{2}\left(\frac{E_{\mathrm{f}}}{E_{\mathrm{i}}}\right)^{1 / 2}\left|M_{\mathrm{c}}\right|^{2}\left|M_{\mathrm{c}}^{*}\right|^{2}, \\
C=\sum_{\mathrm{i}, \mathrm{f}} g\left(\varepsilon_{\mathrm{i}}\right) \sum_{j}\left\langle i\left|\exp \left(i \Delta \bar{K} \cdot \bar{R}_{L}\right)\right| f\right\rangle\left\langle f\left|\exp \left(i \bar{K}_{\mathrm{f}} \cdot \bar{R}_{L}\right)\right| j\right\rangle\left\langle j\left|\exp \left(i \bar{K}_{\mathrm{i}} \cdot \bar{R}_{L}\right)\right| i\right\rangle \exp \left[i\left(\varepsilon_{\mathrm{f}}-\varepsilon_{j}\right) \frac{t}{h}\right] \exp \left[i\left(\varepsilon_{\mathrm{i}}-\varepsilon_{\mathrm{f}}\right) \frac{T}{h}\right], \\
\Delta E=E_{\mathrm{f}}-E_{0} .
\end{gathered}
$$

Using the following equations :

$$
\exp \left[\mp i \varepsilon_{n} \frac{t}{h}\right]|n\rangle=\exp \left[\mp i \mathrm{H}_{\mathrm{A}} \frac{t}{h}\right]|n\rangle, \quad \sum_{n}|n\rangle\langle n|=I,
$$

for $n=i, j$ or $f$, where $\mathrm{H}_{\mathrm{A}}$ is the Hamiltonian of the atomic system, equation (17) becomes :

$$
\begin{aligned}
C & =\sum_{i} g\left(\varepsilon_{\mathrm{i}}\right)\left\langle i\left|\exp \left[i \Delta \bar{K} \cdot \bar{R}_{L}(T)\right] \exp \left[-i \bar{K}_{\mathrm{f}} \cdot \bar{R}_{L}(t)\right] \exp \left(i \bar{K}_{\mathrm{i}} \cdot \bar{R}_{L}\right)\right| i\right\rangle \\
& =\left\langle\exp \left[i \Delta \bar{K} \cdot \bar{R}_{L}(T)\right] \exp \left[-i \bar{K}_{\mathrm{f}} \cdot \bar{R}_{L}(t)\right] \exp \left(i \bar{K}_{\mathrm{i}} \cdot \bar{R}_{L}\right)\right\rangle_{\mathrm{T}},
\end{aligned}
$$

where $R(t)$ and $R(T)$ are the Heisenberg operators and $\langle\ldots\rangle_{\mathrm{T}}$ denotes the thermal average. Substituting this into equation (15) we get :

$$
\begin{aligned}
\frac{\mathrm{d}^{2} \sigma_{\text {int }}}{\mathrm{d} \epsilon \mathrm{d} \Omega}=Q \operatorname{Re} i \int_{0}^{\infty} \frac{\mathrm{d} t}{\hbar} \exp \left[i\left(\Delta E+i \frac{\Gamma}{2}\right) \frac{t}{\hbar}\right] \int_{-\infty}^{+\infty} \frac{\mathrm{d} T}{\hbar} \\
\\
\times \exp \left(-i \epsilon \frac{T}{\hbar}\right)\left\langle\exp \left[i \Delta \bar{K} \cdot \bar{R}_{L}(T)\right] \exp \left[-i \bar{K}_{\mathrm{f}} \cdot \bar{R}_{L}(t)\right] \exp \left(i \bar{K}_{\mathrm{i}} \cdot \bar{R}_{L}\right)\right\rangle_{\mathrm{T}} \cdot
\end{aligned}
$$


Equation (21) gives the basic result for interference scattering from the $L$ th atom. It shows the interference scattering broadening is determined by a three-point correlation function. For an ideal gas the three-point correlation function can be written in the following form:

$$
C=\exp \left[-\frac{1}{2 M_{L} \beta}\left(\bar{K}_{\mathrm{f}} t-\Delta \bar{K} T\right)^{2}-i\left(\frac{\hbar \Delta K^{2}}{2 M_{L}} T+\frac{\hbar \bar{K}_{\mathrm{i}} \cdot \bar{K}_{\mathrm{f}}}{M_{L}} t-\frac{\hbar K_{f}^{2}}{2 M_{L}} t\right)\right],
$$

where $\beta^{-1}$ is the temperature in (eV).

\section{INTERFERENCE SCATTERING OF NEUTRONS BY HARMONIC CRYSTALS}

In this model, the atoms are assumed to undergo harmonic oscillations about the equilibrium positions. The position of the $L$ th atom in the $j$ th unit cell at any time is :

$$
\bar{R}_{j L}(t)=\bar{\rho}_{j}+\bar{r}_{j L}+\bar{\mu}_{j L}(t)
$$

where the position of the $j$ th unit cell is:

$$
\bar{\rho}_{j}=j_{1} \bar{d}_{1}+j_{2} \bar{d}_{2}+j_{3} \bar{d}_{3}
$$

where $j_{1}, j_{2}$ and $j_{3}=0, \mp 1, \mp 2, \ldots$ and $d_{i}$ are the basis vectors of the lattice. $\bar{r}_{j L}$ is the equilibrium position of the $L$ th atom, and $\bar{\mu}_{j L}(t)$ is the displacement of the $L$ th atom from equilibrium (see Maradudin et al., 1963). The atomic displacements are expressed as a superposition of the normal-mode oscillations :

$$
\bar{\mu}_{j L}(t)=\sum_{\bar{q} \lambda}\left(\frac{\hbar}{2 N M_{L} \omega_{\lambda}}\right)^{1 / 2}\left[\mathbf{e}_{\bar{q} \lambda}^{L} a_{\bar{q} \lambda} \exp \left(i \bar{q} \cdot \bar{\rho}_{j}-i \omega_{\lambda} t\right)+\mathbf{e}_{\bar{q} \lambda}^{L *} \exp \left(-i \bar{q} \cdot \bar{\rho}_{j}+i \omega_{\lambda} t\right)\right]
$$

where $\mathbf{e}_{\bar{q} \lambda}^{L}$ is the polarization vector ( $\lambda$ is the vibration branch for the wave vector $\bar{q}$ ) $a_{\bar{q}}$ and $a_{\tilde{q}}^{+}$are the lowering and raising operators, $\omega_{2}$ is the normal mode frequency and $N$ is the number of unit cells. The polarization vectors satisfy the orthogonality relation:

$$
\sum_{L} \mathbf{e}_{\tilde{q} \lambda}^{L^{*}} \cdot \mathbf{e}_{\bar{q} \lambda^{\prime}}^{L}=\delta_{\lambda \lambda^{*}}
$$

In order to evaluate the interference scattering cross section [equation (21)] for the harmonic model of a crystal lattice, we first need to evaluate the thermal average given in equation (20):

$$
C=\left\langle\exp \left[i \Delta \bar{K} \cdot \bar{R}_{L}(T)\right] \exp \left[-i \bar{K}_{\mathrm{f}} \cdot \bar{R}_{L}(t)\right] \exp \left(i \bar{K}_{\mathrm{i}} \cdot \bar{R}_{L}\right)\right\rangle_{\mathrm{T}}
$$

using the following two identities:

$$
\begin{gathered}
\mathrm{e}^{A} \mathrm{e}^{B} \mathrm{e}^{C}=\exp \left\{A+B+C+\frac{1}{2}[A, B]+\frac{1}{2}[A, C]+\frac{1}{2}[B, C]\right\}, \\
\left\langle\mathrm{e}^{\mathrm{Q}}\right\rangle_{\mathrm{T}}=\exp \left[\frac{1}{2}\left\langle Q^{2}\right\rangle_{\mathrm{T}}\right],
\end{gathered}
$$

where the double and higher commutators are zero for harmonic oscillator coordinates, the thermal average can be written as:

$$
\begin{array}{r}
\left\langle\exp \left[i \Delta \bar{K} \cdot \bar{R}_{L}(T)\right] \exp \left[-i \bar{K}_{\mathrm{f}} \cdot \bar{R}_{L}(t)\right] \exp \left(i \bar{K}_{\mathrm{i}} \cdot \bar{R}_{L}\right)\right\rangle_{\mathrm{T}}=\exp \left\{\frac{1}{2}\left[\Delta \bar{K} \cdot \bar{R}(T), \bar{K}_{\mathrm{f}} \cdot \bar{R}_{L}(t)\right]-\frac{1}{2}\left[\Delta \bar{K} \cdot \bar{R}_{L}(T), \bar{K}_{\mathrm{i}} \cdot \bar{R}_{L}(t)\right]\right. \\
\left.+\frac{1}{2}\left[\bar{K}_{\mathrm{f}} \cdot \bar{R}_{L}(t), \bar{K}_{\mathrm{i}} \cdot \bar{R}_{L}\right]-\frac{1}{2}\left\langle\left[\Delta \bar{K} \cdot \bar{R}_{L}(T)-\bar{K}_{\mathrm{f}} \cdot \bar{R}_{L}(t)+\bar{K}_{\mathrm{i}} \cdot \bar{R}_{L}\right]^{2}\right\rangle_{\mathrm{T}}\right\} . \quad(29)
\end{array}
$$

Now we can calculate each term on the RHS of equation (29) using equations (23), (25), (26) and the following relation:

$$
\left\langle a^{+} a\right\rangle_{\mathrm{T}}+\left\langle a a^{+}\right\rangle_{\mathrm{T}}=\operatorname{coth} \frac{\beta \epsilon}{2},
$$




$$
\begin{aligned}
& C=\exp \left\{-\sum_{\bar{q} \lambda} \frac{\hbar}{4 N M_{t} \omega_{\lambda}}\left\{\left[\left(\bar{K}_{\mathrm{i}} \cdot \mathbf{e}_{\bar{q} \lambda}^{L}\right)\left(\bar{K}_{\mathrm{i}} \cdot \mathbf{e}_{\bar{q} \lambda}^{L^{*}}\right)+\left(\bar{K}_{\mathrm{f}} \cdot \mathbf{e}_{\bar{q} \lambda}^{L}\right)\left(\bar{K}_{\mathrm{f}} \cdot \mathbf{e}_{\bar{q} \lambda}^{L^{*}}\right)-\left(\bar{K}_{\mathrm{i}} \cdot \mathbf{e}_{\bar{q} \lambda}^{L}\right)\left(\bar{K}_{\mathrm{f}} \cdot \mathbf{e}_{\bar{q} \lambda}^{L}\right)\right]\right.\right. \\
& \times\left(\mathrm{e}^{-\beta \epsilon / 2}+\mathrm{e}^{\beta \epsilon / 2}\right)-\left(\bar{K}_{\mathrm{i}} \cdot \mathbf{e}_{\bar{q} \lambda}^{L}\right)\left(\bar{K}_{\mathrm{f}} \cdot \mathbf{e}_{\bar{q} \lambda}^{L^{*}}\right) \mathrm{e}^{i \omega_{\lambda} t} \mathrm{e}^{\beta \epsilon / 2}-\left(\bar{K}_{\mathrm{i}} \cdot \mathbf{e}_{\bar{q} \lambda}^{L^{*}}\right)\left(\bar{K}_{\mathrm{f}} \cdot \mathbf{e}_{\bar{q} \lambda}^{L}\right) \mathrm{e}^{-i \omega_{\lambda} t} \mathrm{e}^{-\beta \epsilon / 2} \\
& +\left(\Delta \bar{K} \cdot \mathbf{e}_{\tilde{q} \lambda}^{L *}\right)\left(\bar{K}_{\mathrm{i}} \cdot \mathbf{e}_{\bar{q} \lambda}^{L}\right) \mathrm{e}^{i \omega_{\lambda} T} \mathrm{e}^{-\beta \epsilon / 2}+\left(\Delta \bar{K} \cdot \mathbf{e}_{\bar{q} \lambda}^{L}\right)\left(\bar{K}_{\mathrm{i}} \cdot \mathbf{e}_{\tilde{q} \lambda}^{L *}\right) \mathrm{e}^{-i \omega_{\lambda} T} \mathrm{e}^{\beta \epsilon / 2} \\
& \left.\left.-\left(\bar{K}_{\mathrm{f}} \cdot \mathbf{e}_{\tilde{q} \lambda}^{L}\right)\left(\Delta \bar{K} \cdot \mathbf{e}_{\bar{q}_{\lambda}}^{L *}\right) \mathrm{e}^{i \omega_{\lambda}(T-t)} \mathrm{e}^{-\beta \epsilon / 2}-\left(\bar{K}_{\mathrm{f}} \cdot \mathbf{e}_{\bar{q} \lambda}^{L *}\right)\left(\Delta \bar{K} \cdot \mathbf{e}_{\bar{q} \lambda}^{L}\right) \mathrm{e}^{-i \omega_{\lambda}(T-t)} \mathrm{e}^{\beta \varepsilon / 2}\right\} \operatorname{csch}\left(\frac{\beta \epsilon}{2}\right)\right\} .
\end{aligned}
$$

Let us define the usual generalized phonon frequency distribution function:

$$
G_{\alpha \alpha_{p}}^{L}(\epsilon)=\frac{1}{N} \sum_{\bar{q} \lambda} \mathbf{e}_{\bar{q} \lambda}^{L} \cdot \mathbf{e}_{\bar{q} \lambda}^{L^{*}} \delta\left[\epsilon-\hbar \omega_{\lambda}(\bar{q})\right], \quad \epsilon>0 .
$$

Using equations (32) and (31) and after some algebra, we get:

$$
C=\exp \left\{\left[z_{L}(t)+z_{L}^{\mathrm{f}, \Delta}(T, t)\right]-\left[z_{L}^{\mathrm{i}, \Delta}(T)+z_{L}^{\mathrm{i}}(0)+z_{L}^{\mathrm{f}}(0)-z_{L}(0)\right]\right\}=\mathrm{e}^{-\lambda(T, t)},
$$

where we defined the following:

$$
\begin{aligned}
& z_{L}^{\mathrm{i}}(0)=\sum_{\alpha \gamma} \frac{\hbar^{2}}{4 M_{L}} \int_{-\infty}^{+\infty} \frac{\mathrm{d} \epsilon}{\epsilon} \bar{K}_{\mathrm{i} \alpha} \cdot \bar{K}_{\mathrm{i} \gamma} G_{\alpha \gamma}^{L}(\epsilon) \mathrm{e}^{\beta \epsilon / 2} \operatorname{csch} \frac{\beta \epsilon}{2}, \\
& z_{L}^{\mathrm{f}}(0)=\sum_{\alpha \gamma} \frac{\hbar^{2}}{4 M_{L}} \int_{-\infty}^{+\infty} \frac{\mathrm{d} \epsilon}{\epsilon} \bar{K}_{\mathrm{fa}} \cdot \bar{K}_{f \gamma} G_{\alpha \gamma}^{L}(\epsilon) \mathrm{e}^{\beta \epsilon / 2} \operatorname{csch} \frac{\beta \epsilon}{2}, \\
& z_{L}(0)=\sum_{\alpha \gamma} \frac{\hbar^{2}}{4 M_{L}} \int_{-\infty}^{+\infty} \frac{\mathrm{d} \epsilon}{\epsilon} \bar{K}_{\mathrm{i} \alpha} \cdot \bar{K}_{f_{\gamma}} G_{\alpha \gamma}^{L}(\epsilon) \mathrm{e}^{\beta c / 2} \operatorname{csch} \frac{\beta \epsilon}{2}, \\
& z_{L}(t)=\sum_{\alpha \gamma} \frac{\hbar^{2}}{4 M_{L}} \int_{-\infty}^{+\infty} \frac{\mathrm{d} \epsilon}{\epsilon} \bar{K}_{\mathrm{i} \alpha} \cdot \bar{K}_{\mathrm{fy}} G_{\alpha \gamma}^{L}(\epsilon) \mathrm{e}^{\beta \epsilon / 2} \mathrm{e}^{-i \epsilon(t / \hbar)} \operatorname{csch} \frac{\beta \epsilon}{2}, \\
& z_{\dot{L}}^{\mathrm{i}, \Delta}(T)=\sum_{\alpha \gamma} \frac{\hbar^{2}}{4 M_{L}} \int_{-\infty}^{+\infty} \frac{\mathrm{d} \epsilon}{\epsilon} \bar{K}_{\mathrm{i} \alpha} \cdot \Delta \bar{K}_{\gamma} G_{\alpha \gamma}^{L}(\epsilon) \mathrm{e}^{\beta \epsilon / 2} \mathrm{e}^{-i \epsilon(T / h)} \operatorname{csch} \frac{\beta \epsilon}{2}, \\
& z_{\dot{L}}^{\mathrm{f} \Lambda}(T)=\sum_{\alpha \gamma} \frac{\hbar^{2}}{4 M_{L}} \int_{-\infty}^{+\infty} \frac{\mathrm{d} \epsilon}{\epsilon} \bar{K}_{\mathrm{fa}} \cdot \Delta \bar{K}_{\gamma} G_{\alpha \gamma}^{L}(\epsilon) \mathrm{e}^{\beta \epsilon / 2} \mathrm{e}^{-i \epsilon[(T-t) / \hbar]} \operatorname{csch} \frac{\beta \epsilon}{2}
\end{aligned}
$$

and

$$
\begin{aligned}
& \gamma(T, t)=\int_{0}^{+\infty} \frac{\mathrm{d} \epsilon}{\epsilon} \sum_{\alpha} G_{\alpha \alpha}^{L}(\epsilon)\{[ \frac{\hbar^{2} K_{\mathrm{i}}^{2}}{2 M_{L}}+\frac{\hbar^{2} K_{\mathrm{f}}^{2}}{2 M_{L}}-\frac{\hbar^{2} \bar{K}_{\mathrm{i}} \cdot \bar{K}_{\mathrm{f}}}{2 M_{L}}-\frac{\hbar^{2} \bar{K}_{\mathrm{i}} \cdot \bar{K}_{\mathrm{f}}}{2 M_{L}} \cos \frac{\epsilon t}{\hbar} \\
&\left.+\frac{\hbar^{2} \bar{K}_{\mathrm{i}} \cdot \Delta \bar{K}}{2 M_{L}} \cos \frac{\epsilon T}{\hbar}-\frac{\hbar^{2} \bar{K}_{\mathrm{f}} \cdot \Delta \bar{K}}{2 M_{L}} \cos \frac{\epsilon(T-t)}{\hbar}\right] \operatorname{coth} \frac{\beta \epsilon}{2} \\
&\left.+i\left[\frac{\hbar^{2} \bar{K}_{\mathrm{i}} \cdot \bar{K}_{\mathrm{f}}}{2 M_{L}} \sin \frac{\epsilon t}{\hbar}-\frac{\hbar^{2} \bar{K}_{\mathrm{i}} \cdot \Delta \bar{K}}{2 M_{L}} \sin \frac{\epsilon T}{\hbar}+\frac{\hbar^{2} \bar{K}_{\mathrm{f}} \cdot \Delta \bar{K}}{2 M_{L}} \sin \frac{\epsilon(T-t)}{\hbar}\right]\right\} .
\end{aligned}
$$

For short collision times and for crystals with cubic symmetry, we can expand $\gamma(T, t)$ as:

$$
\mathrm{e}^{-\gamma(T, t)}=\exp \left[\frac{-1}{2 M_{L} \beta_{\mathrm{eff}}}\left(\bar{K}_{\mathrm{f}} t-\Delta \bar{K} T\right)^{2}-i\left(\frac{\hbar \Delta K^{2}}{2 M_{L}} T+\frac{\hbar \bar{K}_{\mathrm{i}} \cdot \bar{K}_{\mathrm{f}}}{M_{L}} t-\frac{\hbar K_{\mathrm{f}}^{2}}{2 M_{L}} t\right)\right]
$$

where 


$$
\beta_{\text {eff }}^{-1}=\frac{1}{2} \int_{0}^{\infty} \epsilon \mathrm{d} \epsilon \operatorname{coth} \frac{\epsilon \beta}{2} \sum_{\alpha} G_{\alpha x}^{L}(\epsilon)
$$

and $\beta^{-1}$ is the real temperature in energy units.

Equation (41) is identical in form to equation (22) which has been derived for an ideal gas. However, equation (41) has an effective temperature. Based on this, it is clear that the interference scattering can be treated using an ideal gas result with effective temperature. $\beta_{\text {erf }}^{-1}$ is the usual effective temperature in energy units determined by the crystal phonon spectrum, as has been previously defined by Lamb (1939), Koppel and Houston (1968) for resonant absorption, and Shamaoun and Summerfield (1990) for resonant scattering.

\section{CONCLUSIONS}

For resonant neutron processes, the interference differential scattering cross section can be written in terms of a three-point correlation function. This function was evaluated for ideal gas and harmonic crystal models. It was shown that for short collision times the expansion of $\gamma(T, t)$ gave a similar result to the ideal gas (22). However, the harmonic crystal result has an effective temperature (which is the same as the one that was found in resonant scattering and absorption). Therefore, the interference scattering cross section can be treated using the ideal gas result with effective temperature. What this also means is that there is no anomalous chemical binding effect for the interference scattering to explain the unusual results found in some earlier experiments (Tsang and Brugger, 1979, 1980).

\section{REFERENCES}

Bethe H. and Placzek G. (1937) Phys. Rev. 51, 450.

Breit G. and Wigner E. (1936) Phys. Rev. 49, 519.

Koppel J. and Houston D. (1968) U.S. Atomic Energy Commission, Report GA-8774.

Kuwaifi A., Summerfield G. and Shamaoun A. (1990) Ann. nucl. Energy 17, 565.

Lamb W. E. (1939) Phys. Rev. 55, 190.

Lovesey S. and Springer T. (Eds) (1977) Dynamics of Solids and Liquids by Neutron Scattering. Springer-Verlag, New York.

Maradudin A., Montroll E. and Weiss G. (1963) Theory of Lattice Dynamics in the Harmonic Approximation. Academic Press, New York.

Osborn R. K. and Yip S. (1966) The Foundations of Neutron Transport Theory. Gordon \& Breach, New York.

Placzek G. and Van Hove L. (1955) Nuovo Cim. 1, 233.

Shamaoun A. and Summerfield G. (1990) Ann. nucl. Energy 17, 229.

Trammell G. T. (1984) Condensed Matter Research Using Neutrons, Vol. 112 of NATO (Steven W., Lovesey W. and Scherm R., Eds). Plenum, New York.

Tsang F. and Brugger R. (1979) Nucl. Sci. Engng 72, 52.

Tsang F. and Brugger R. (1980) Nucl. Sci. Engng 74, 34.

Van Hove L. (1954) Phys. Rev. 95, $249,1374$.

Word R. E. and Trammell G. T. (1981) Phys. Rev. B24, 2430. 